\title{
Found in Translation
}

\author{
By Jack M. Gorman, MD
}

Investigation of central nervous system (CNS) diseases has always been hampered by the relative inaccessibility of the brain compared with other organs and a lack of animal models. Whereas reasonably good animal models of cardiovascular, gastrointestinal, and endocrine diseases exist (dogs get heart attacks, ulcers, and diabetes), animal models for multiple sclerosis (MS), Parkinson's disease, and Alzheimer's disease often leave investigators feeling as if they are dealing with replicas of human disease that are only partially accurate. Worse, it is difficult to conceive of an animal model for schizophrenia, bipolar disorder, or anorexia nervosa. Without such models, however, it is a challenge to advances in the field of biomedical investigation.

Yet strides have been made in developing animal models of neurological and psychiatric diseases that have furthered knowledge of pathophysiology and therapeutics. It is important to remember that at the end of the day an animal model does not have to be an exact simulacrum of a human disorder; it simply has to be useful in developing a better understanding of underlying biological processes that lead to efficacious treatments. Is experimental autoimmune encephalitis an adequate representation of MS? Probably not, but it is a useful animal model if it allows researchers to screen and test drugs that ultimately prove effective in treating MS in humans. Watching rats poke their heads out of an enclosed space in the plus maze apparatus may not strike us as the same as observing a patient with an anxiety disorder, but this test has enabled us to learn more about the mechanism of action of antidepressant drugs. Furthermore, many drugs that enable rats to tolerate the open arms of the plus maze for longer periods of time turn out to be effective anxiolytics in humans.

An important role of animal models for CNS disease has become the evaluation of endophenotypes. That is, certain features of an illness may be more amenable to animal modeling than the entire illness taken as whole. Finding an animal model for schizophrenia, for example, may be difficult, but one aspect of schizophrenia-impaired pre-pulse inhibition-can indeed by demonstrated in rodents and monkeys. Thus, researchers have shown that pre-pulse inhibition abnormalities can be induced in rodents by administration of drugs that cause psychosis in humans; antipsychotic drugs normalize the pre-pulse inhibition defect in rodents. This permits the consideration of an animal model of an endophenotype of schizophrenia, abnormal pre-pulse inhibition, as a window on one portion of the pathology in the human disorder. Molecular and genetic studies can then be carried out in the animal model, which may in turn prove useful in finding genes that are abnormally regulated in schizophrenia.

This issue of CNS Spectrums provides an important insight into the use of animal models to study neurological and psychiatric illness. Animal models of Alzheimer's disease, obsessive-compulsive disorder, Parkinson's disease, and depression are presented. The articles represent three key goals of this journal. First, they are attempts to educate clinicians about cutting-edge science that will ultimately influence how physicians treat patients. Second, we combine psychiatric and neurological diseases, demonstrating that both specialties benefit from reviewing each speciality's target disorders. Third, the articles are written by an international group of authors. We hope you will feel that even in your office you are connected to a world of neuroscience that is rapidly changing our understanding of the brain and brain disorders.

This month we introduce a new column by an esteemed colleague and friend, David Dunner, MD, FACP, professor of psychiatry at the University of Washington. In his new column, Dr. Dunner will present an outline of a case and then pose questions to readers. We will then publish your responses in a subsequent issue of CNS Spectrums. This interactive feature will be stimulating and thought-provoking. I would expect nothing else from Dr. Dunner, whom I first met many years ago when we both worked at Columbia University. Dr. Dunner had already made seminal contributions to the treatment of bipolar disorder at that time and was (and remains) a superb teacher. He has continued to be a major contributor to the scientific literature on mood disorders. We hope you enjoy his new column, we and eagerly await your correspondence. CNS

Dr. Gorman is the editor of this journal and Esther and Joseph Klingenstein Professor of Psychiatry and chair of the Department of Psychiatry at Mount Sinai School of Medicine in New York City. 